GlyOBz (VI) und -AlaOBz (VII) wurden aus Äthanol, Z-Nitroarg-serOÄt (VIII) und -SerOBz (IX) aus wasserhaltigem Aceton (Aceton : $\mathrm{H}_{2} \mathrm{O}=9: 1$ ) umkristallisiert.

Die Z-Peptidester sind in Tab. 1 aufgeführt.

Die Darstellung der Aminosäurebenzylester erfolgte nach Miller und $\mathrm{W}_{\mathrm{AELSCH}}{ }^{23}$. Glycin- und Alaninbenzylester wurden als Benzolsulfonate (unter Zugabe einer äquivalenten Menge Triäthylamin) in die Peptidsynthese eingesetzt. Das Serinbenzylester-benzolsulfonat wurde wie unter $1 c$ beschrieben in das Hydrochlorid verwandelt.
Zur Hydrierung der Z-Peptidbenzylester wurde verfahren wie unter $2 c$ beschrieben. L-Arginyl-DL-alaninacetat (XII) wurde aus Methanol-Äthanol-Äther umkristallisiert. L-Arginyl-DL-seryl-acetat (XIII) wurde in amorpher Form erhalten und mit überschüssiger Flaviansäure aus wäßriger Lösung als kristallines Diflavianat gefällt.

Die Peptide sind in Tab. 2 aufgeführt.

Wir danken der Deutschen Forschungs gemeins chaft und dem $\mathrm{Ch}$ emiefonds für die Unterstützung der Arbeit.

\title{
Konstitution und Wirkung biologisch aktiver Verbindungen
}

\author{
IV. Mitt.: Weitere halogenhaltige, phosphorfreie organische Insektizide \\ Von Werner Perkow \\ Aus dem Forschungslaboratorium der Norddeutschen Affinerie, Hamburg \\ (Z. Naturforschg. 12 b, 92-95 [1957]; eingegangen am 21. August 1956)
}

\begin{abstract}
Als „biologisch aktive Zentren“ werden bei weiteren chlorierten Kohlenwasserstoffen und anderen phosphorfreien Insektiziden wiederum Kohlenstoffatome mit charakteristisch verminderter Elektronendichte für die Wechselwirkung mit Fermenten verantwortlich gemacht. Das gleiche Wirkungsprinzip tritt auch in anderen Verbindungsklassen auf.
\end{abstract}

In vorangegangenen Mitteilungen versuchten wir, bei der Betrachtung der insektizid wirkenden Verbindungen Dichlordiphenyltrichloräthan (DDT) ${ }^{1}$, Diäthyl-p-nitrophenyl-thiophosphat (E 605 $)^{2}$ und weiterer phosphorhaltiger Insektengifte ${ }^{3}$ zu zeigen, daß jeweils eine charakteristische Ladungsverteilung in den Molekülen mit der biologischen Wirksamkeit in Zusammenhang steht. Wir sind der Ansicht, daß stets nur ein begrenzter Molekülteil oder oft auch nur ein einziges, besonders beschaffenes Atom als Wirkungsträger anzusehen ist. Die an einem solchen „biologisch aktiven Zentrum“ vorhandene Elektronenanordnung tritt bei allen Gliedern einer biologisch wirksamen Gruppe mit gleichem Grundgerüst auf. Beispielsweise bewirken bei den der DDT-Familie zugehörigen Verbindungen die Substituenten des mittelständigen, tertiären Kohlenstoffatoms an diesem stets eine stark verringerte Elektronendichte:

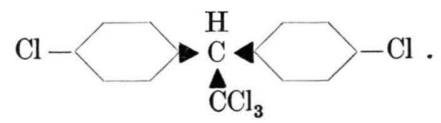

Eine Stärkung oder Schwächung dieses charakteristischen Zustandes hat bei der DDT-Gruppe ebenso wie bei anderen Substanzgruppen stets auch eine entsprechende Änderung der insektiziden WirkungsIntensität zur Folge. Man darf daraus den Schluß ziehen, daß bei Abwandlungen eines als biologisch wirksam erkannten Moleküls die typischen Eigenarten seines „biologisch aktiven Zentrums“ erhalten bleiben müssen oder innerhalb gewisser Grenzen möglichst noch intensiviert werden sollen.

Der Wirkungs-Mechanismus aller Insektizide dürfte auf einer Wechselwirkung mit Fermenten beruhen und kann als energetisches Wechselspiel zwischen komplementär beschaffenen Molekülgefügen aufgefaßt werden. Das „aktive Zentrum“ bildet die Grundlage dieser Wechselwirkung. Die Beschaffenheit der übrigen Molekülteile liefert gewissermaßen die Feinstruktur des Schlüssels in dem bekannten, bildhaften Vergleich des Wirkungsprinzips der Fermentreaktionen mit dem Formverhältnis von Schlüssel zu Schloß. Das Gefüge der Reaktionspartner muß sich sowohl in räumlicher wie auch energetischer Form an den Wirkungspunkten entsprechen. Eine Änderung der Feinstruktur durch geringfügige Variation einzelner Molekülteile bei erhaltener typischer Ladungsverteilung kann z. B. eine Verschie-

3 W. PERKow, Z. Naturforschg. 12 b, 33 [1957].

1 W. Perkow, Z. Naturforschg. 11 b, 389 [1956].

2 W. Perkow, Z. Naturforschg. 11 b, 460 [1956]. 
bung im „Wirkungsspektrum“ der Insektizide zur Folge haben. Ihre Giftwirkung verlagert sich, wodurch einzelne Schädlingsarten nicht mehr, andere dagegen neu betroffen werden. Die arteigene, spezifische Natur der Fermente kann bei einem derartigen Wechsel ebenso die Ursache sein wie eine Änderung der chemisch-physikalischen Eigenschaften des Giftstoffs, die sich auf die Durchdringungsfähigkeit der Epicuticula, die Zellpermeabilität und andere wichtige Faktoren auswirkt.

Wir wollen unter diesen Gesichtspunkten nochmals einige entferntere Verwandte der DDT-Familie betrachten, deren früher genannte Glieder alle eine insektizide Wirkung ausüben, ohne indessen z. B. auf Spinnmilben stark toxisch zu wirken. - Ersetzen wir im DDT-Gerüst das Wasserstoffatom des tertiären Kohlenstoffs durch eine Hydroxylgruppe, so bleibt die verringerte Elektronendichte am „aktiven Zentrum" und damit das Wirkungsprinzip eines Angriffs auf das Nervensystem der Tiere erhalten. Das "Wirkungsspektrum“ jedoch verschiebt sich durch diesen Eingriff: Das entstandene Di (p-chlorphenyl)trichloräthanol (I)

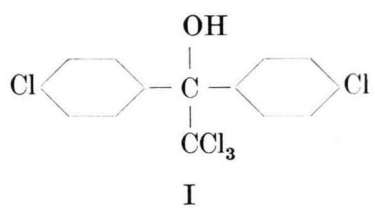

ist ein spezifisch auf Spinnmilben wirkendes „Akarizid" ohne einen nennenswerten Effekt auf die durch die Muttersubstanz DDT betroffenen Insekten. Die Hydroxylgruppe scheint mit dieser spezifischen Wirkung in engerem Zusammenhang zu stehen, denn sie ist auch in den akarizid wirksamen Verbindungen 4.4-Dichlorbenzilsäureäthylester (II), $\mathrm{Di}(p$ chlorphenyl)-äthanol (III) und $p$-Chlorphenyl-trichlormethyl-carbinol (IV) enthalten.

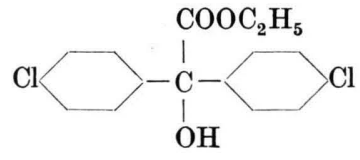

II

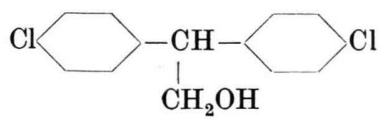

III

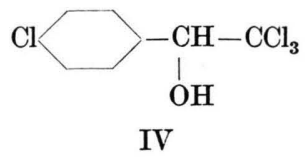

Als Akarizid begegnet uns auch wieder das mit dem DDT nah verwandte $\mathrm{Di}$ ( $p$-chlorphenyl) -trifluoräthan
(V), von dem wir früher bereits berichteten, daß es offenbar durch seine gegenüber dem DDT stark verringerte Lipoidlöslichkeit die insektiziden Eigenschaften eingebüßt hat.

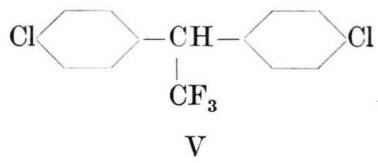

Das bei all diesen Verbindungen gleichartig auftretende biologische Zentrum finden wir - ebenfalls durch $p$-Chlorphenylgruppen induktiv hervorgerufen - in den akarizid wirksamen Verbindungen $\operatorname{Di}(p$-chlorphenyl) -methan (VI), $p$-Chlorbenzyl- $p$ chlorphenyläther (VII) und $\operatorname{Di}(p$-chlorphenoxy)methan (VIII) :
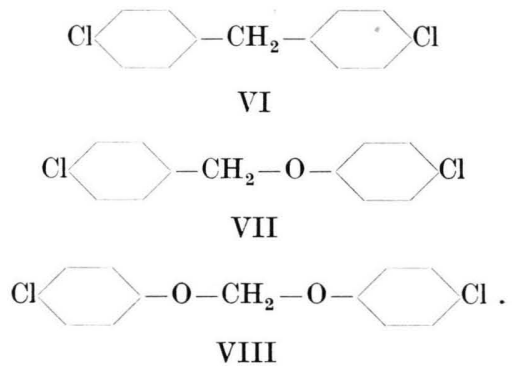

Bei den beiden Ätherverbindungen wird man bei der Betrachtung der Ladungsverteilung nicht die mesomere, beim DDT gebrauchte Formulierung zu Hilfe nehmen, sondern den elektronen-anziehenden, durch den $p$-Chlor-Substituenten verstärkten induktiven Effekt der Phenoxygruppe. Daß ein von zwei Seiten her gleichzeitig ausgeübter Einfluß auf das mittelständige Kohlenstoffatom für die biologische Wirkung maßgeblich ist, mag auch daraus hervorgehen, daß die nahe verwandten Verbindungen $\operatorname{Di}(p$-chlorphenoxy)-äthan (IX) und $\mathrm{Di}(p$-chlorphenoxy)-propan (X) ohne erkennbare biologische Wirkung sind.

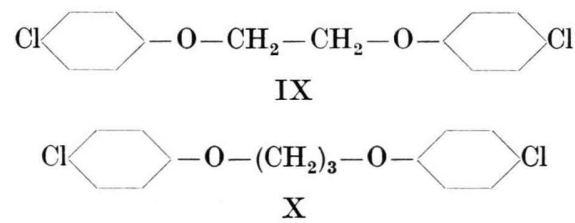

Auch die mehrgliedrigen, chlorierten Ringsysteme, wie Heptachlor (XI) und Chlordan (XII), Aldrin (XIII) und Dieldrin (XIV), lassen charakteristische „biologisch aktive Zentren“ mit wiederum stark verringerter Ladungsdichte erkennen. 

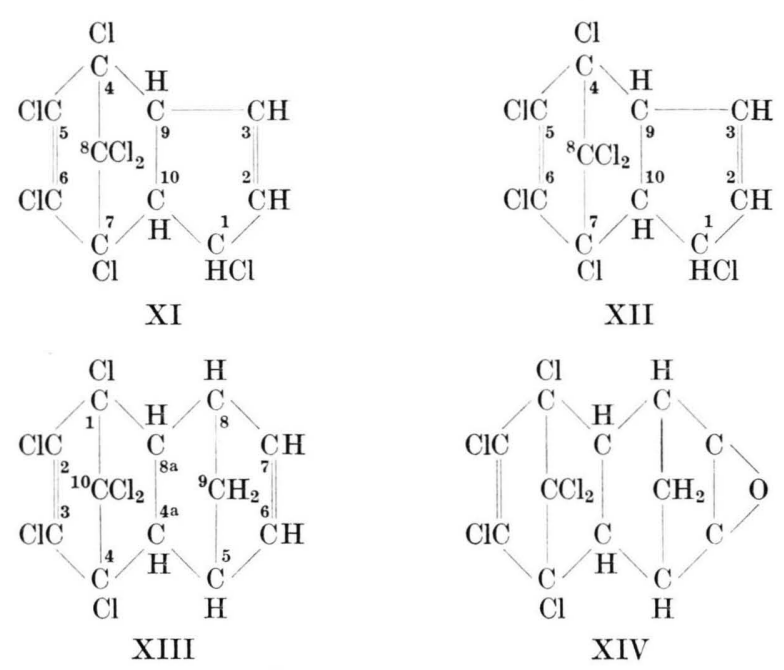

Die Ähnlichkeit in der Elektronen-Konfiguration dieser Insektizidgruppe mit der DDT-Familie ist größer, als es bei oberflächlicher Betrachtung erscheinen mag. In der folgenden Schreibweise der Formeln sind die Kohlenstoffatome, die wir als Ausgangspunkte der biologischen Aktivität betrachten, markiert.
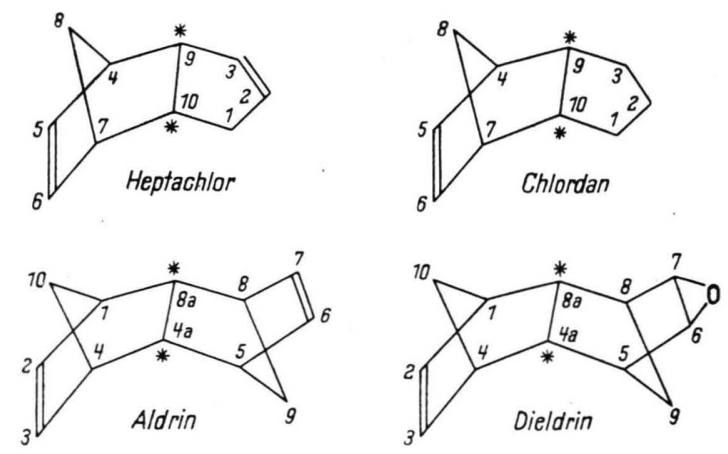

Die beiden Kohlenstoffatome 9 und 10 im Chlordan und Heptachlor werden zunächst durch den im Formelbild linksseitig benachbarten, stark mit Chloratomen substitutierten Ring 4, 5, 6, 7, 8 in gleicher Weise im Sinne eines Elektronensogs beeinflußt, wie es beim DDT durch die beiden $p$-Chlorphenylreste geschieht. Rechtsseitig im Formelbild nimmt das Chloratom am Kohlenstoff 1 des Chlordans oder Heptachlors die gleiche Position gegenüber dem ,,aktiven" C 10 ein, wie die aliphatisch substituierten Chloratome der DDT-Gruppe zum tertiären Kohlenstoff. Die Doppelbindung 2,3 im Heptachlor befindet sich zum C 9 in gleicher Stellung wie diejenige in den ebenfalls insektizid wirksamen $\mathrm{Di}(p$-chlor- phenyl) -propen-Derivaten. Der im Formelbild von Aldrin und Dieldrin rechtsseitig von der Verbindungslinie 4 a, 8 a stehende Molekülteil (an C 5 und C 8 entsprechend umgeklappt zu denken in den stereoisomeren Verbindungen Isodrin und Endrin) läßt zunächst die Möglichkeit eines zweiten „biologisch aktiven " Kohlenstoffpaares 5,8 offen. Wahrscheinlicher ist ein induzierender Einfluß des Ringes $5,6,7,8$, 9, wie es den Verhältnissen der linksseitigen Molekülhälfte annähernd entspricht. Berechnungen der Elektronendichten können diese Überlegun. gen weiter fördern.

Es sind polycyclische, halogenierte Verbindungen bekannt, in denen, nach dem Formelbild zu urteilen, ein ähnlicher induktiver Einfluß auf ein zentral gelagertes Kohlenstoffpaar ausgeübt wird, ohne daß diese Stoffe insektizide Wirkung zeigen. Hier können nur Untersuchungen über die genaue Molekülgestalt, über die Winkelbildung an den angenommenen Wirkungszentren und über die räumliche Beschaffenheit der benachbarten Gruppen Klarheit bringen. Die Stellung der Ausgangspunkte biologischer Aktivität muß sterisch so exponiert sein, daß eine Wechselwirkung mit Fermenten bzw. allgemein mit biologischen Wirkungspartnern im Bereich molekularer energetischer Beziehungen ermöglicht wird. Das stark gewinkelte Fermentmolekül besitzt entsprechende, wahrscheinlich räumlich und energetisch komplementär beschaffene aktive Stellen, die nur für einen spezifisch „passenden“ aktiven Molekülteil des Wirkungspartners zugänglich sind.

Wir sind weiter der Ansicht, daß das durch eine verringerte Elektronendichte gekennzeichnete, also „positivierte“ Kohlenstoffatom bei zahlreichen weiteren Verbindungen im Zusammenhang mit der Wirkung auf das Zentralnerven-System steht. Bei einigen aliphatischen, halogenierten Kohlenwasserstoffen überschneiden sich die insektizide und die beim Warmblüter narkotisierende Wirkung. Chloroform, Tetrachlorkohlenstoff, Trichloräthylen, Hexachloräthan, Dichlorpropan, Dichlorpropen und weitere Substanzen dieser Art zeigen beide Effekte. Sie alle besitzen das übereinstimmende Merkmal eines oder mehrerer durch elektronenaffine Substituenten in ihrer Ladungsdichte geminderter Kohlenstoffatome. Die Halogene können durch andere elektronenaffine Atome oder Gruppen ersetzt oder unterstützt werden. Nitromethan, Trichloracetonitril und Trichlornitromethan (Pikrylchlorid) sind Beispiele derartiger, ebenfalls insektizid wirkender Verbindungen. 
Die Halogenatome sind in diesen Fällen also nicht in erster Linie Wirkungsträger, wie zuweilen vermutet wurde. Sie erfüllen vielmehr zweierlei Funktionen: Einerseits verursachen sie durch ihre Elektronenaffinität jene verringerte Ladungsdichte am Kohlenstoff, die wir als eigentliche Ursache der biologischen Aktivität ansehen. Andererseits erhöhen sie die Lipoidlöslichkeit der Verbindungen und schaffen dadurch eine Voraussetzung für das Vordringen der Verbindungen zum Wirkungsort, also zur lipoidhaltigen Nervensubstanz.

Wir werden auf die Beziehungen zwischen Konstitution und Wirkung zentral erregender und dämp- fender Verbindungen im pharmakologischen Zusammenhang noch ausführlicher zu sprechen kommen. Ferner werden wir später zeigen, daß das Prinzip des „biologisch aktiven Zentrums" in zahlreichen weiteren toxikologisch und pharmakologisch bedeutsamen Verbindungsklassen synthetischen und natürlichen Ursprungs regelmäßig zu finden ist. Auch Mitosegifte und cancerogene Stoffe lassen diese Merkmale erkennen, ebenso Verbindungen mit Wuchsstoffcharakter und solche mit anderer biologischer Aktivität, deren Eigenschaften ebenfalls mit einem Eingriff in fermentative Prozesse der Organismen in Zusammenhang stehen.

\title{
Structure of $\mathrm{Co}(\mathrm{CO})_{4} \mathrm{H}$ and $\mathrm{Fe}(\mathrm{CO})_{4} \mathrm{H}_{2}$
}

\author{
by Andrew D. Liehr \\ Bell Telephone Laboratories, Inc. Murray Hill, New Jersey \\ (Z. Naturforschg. 12 b, 95-96 [1957] ; eingegangen am 19. November 1956)
}

\begin{abstract}
By use of symmetry arguments new structures are proposed for the controversial metal-carbonyl hydrides of iron and cobalt. The proposed structures predict the possibility of a hindered rotation of the bonded hydrogen atoms.
\end{abstract}

The metal-carbonyl hydrides of iron and cobalt have puzzled chemists for a long time. Their physical properties have contradicted most of the previously proposed structures. They are known, by the electron diffraction work of Ewens and Lister ${ }^{1}$, to be pseudo-tetrahedral (that is, to have tetrahedrally arranged carbon monoxide ligands) and to behave as moderately strong acids ${ }^{2}$. Their infrared spectrum exhibits a metal-hydrogen stretch. ing mode ${ }^{3}$, but no carbon-hydrogen or oxygenhydrogen stretching modes ${ }^{3,4}$. And their nuclear magnetic resonance spectrum indicates a large diamagnetic shielding of the proton ${ }^{5}$. All of these experimental observations must be explained by any postulated configuration.

Assuming a tetrahedral arrangement of the carbon monoxide ligands, we propose that the hydro-

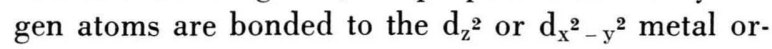

1 R. V. G. Ewens and M. W. Lister, Trans. Faraday Soc. 35, 681 [1939].

2 W. Hieber, Chemie 55, 25 [1942].

3 W. F. Edgell, C. Magee, and G. Gallop, J. Amer. chem. Soc. 78, 4185 [1956].

4 R. A. Friedel, I. Wender, S. L. Shufler, and H. W. SternBERG, J. Amer. chem. Soc. 77, 3951 [1955].

5 H. S. Gutowsky as quoted in Reference 4; F. A. Cotron and G. Wilkinson, Chem. and Ind. 44, 1305 [1956]. bitals in the case of cobalt and to both the $\mathrm{d}_{\mathrm{z}}{ }^{2}$ and

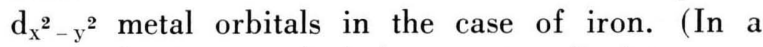
system having tetrahedral symmetry [point group $T_{d}$ ] the degenerate $d_{z}{ }^{2}$ and $d_{x}{ }^{2}-y^{2}$ orbitals of iron and cobalt [symmetry E] are nonbonding as far as the carbon monoxide ligands are concerned ${ }^{6}$; however, they are bonding for the attached hydrogen atoms.) This bonding scheme implies that the hydrogen atoms are located on the four-fold rotationreflection axes of the metal-carbon monoxide tetrahedron in the region between the metal atom and the carbon monoxide ligands (see Fig. $1 \mathrm{a}$ and $1 \mathrm{~b}$ ) The proposed electronic configuration (isoelectronic with $\left.\mathrm{Ni}(\mathrm{CO})_{4}\right)$ may be pictorially described as a "pseudo-nickel carbonyl" configuration ${ }^{2}$.

The bonding proposed in this note for $\mathrm{Co}(\mathrm{CO})_{4} \mathrm{H}$ and $\mathrm{Fe}(\mathrm{CO})_{4} \mathrm{H}_{2}$ is the same as that needed to explain the properties of $\left(\mathrm{C}_{5} \mathrm{H}_{5}\right)(\mathrm{Cr}, \mathrm{Mo}, \mathrm{W})$

6 The latter statement is rigorously true only if metal-carbonyl $\sigma$-bonding is assumed to predominate over metalcarbonyl $\pi$-bonding. See J. H. VAn Vleck, J. chem. Physics. 3, 803 [1935].

7 A. D. Liehr, Naturwissenschaften, in press.

8 E. O. Fischer, W. Hafner, and H. O. Stahl- Z. anorg. u. allg. Chem. 282, 47 [1955].

- T. S. Piper and G. Wilkinson, J. Inorg. Nuc. Chem. 3, 104 [1956]. 